\title{
Wire-arc additive manufacturing of Al-Mg alloy using CMT and PMC technologies
}

\author{
Bianca F. Gomes ${ }^{1,2,}{ }^{*}$, Paulo J. Morais ${ }^{1}$, Vitor Ferreira ${ }^{1}$, Margarida Pinto $^{1}$, and Luiz H. de \\ Almeida $^{2}$ \\ ${ }^{1}$ Instituto de Soldadura e Qualidade, 2740-120 Porto Salvo, Portugal \\ ${ }^{2}$ Universidade Federal do Rio de Janeiro, PEMM, 21941-972 Rio de Janeiro, RJ, Brasil
}

\begin{abstract}
Among the several metallic additive manufacturing (MAM) technologies available, the wire-and-arc based ones are very beneficial due to the lower operational costs, higher efficiency use of raw materials, and high deposition rates achieved. The Cold Metal Transfer (CMT) process stands out by the lower heat input compared to the other wire-and-arc based methods. On the other hand, processes such as Pulse Multi Control (PMC) and its variants have not been tested yet in additive manufacturing and for this reason they should be evaluated. Therefore, considering the technologies potential and the need of automotive and aeronautical industry of manufacturing parts of complex and optimized geometry in a faster way, the study of these technologies is very relevant. Thus, the objective of this paper is the additive manufacturing of walls with Al-Mg alloy using CMT, CMT-Pulse, PMC, PMC-Mix, and MIG-Pulse, and the evaluation of the hardness, mechanical strength, and porosity of the manufactured parts aiming future industrial applications. The results showed good mechanical properties, small pore fraction, and geometric uniformity of parts produced with PMC and PMC-Mix. MIG-Pulse and PMC parts presented the smaller pore fraction among the GMAW variants, although no difference was noticed in the mechanical properties of the parts.
\end{abstract}

\section{Introduction}

The metal additive manufacturing (MAM) has stood out for numerous advantages such as less material waste, being able to produce customized parts, and allow the manufacturing of complex shape and topology optimized parts when compared to traditional subtractive manufacturing. One distinct fact is that the complex parts manufacturing not implies higher processing costs and also MAM might reduce the weight and lead time of the parts [1]. Among the MAM technologies available there are the wire arc additive manufacturing (WAAM) ones. The wire-arc based equipment is preferable when producing large components [2-4], due to the higher deposition rates, even 10 times higher than the laserbased or electron beam-based processes. Furthermore, the wire as feedstock is a low-cost, more efficient and cleaner option than the powder-based equipment $[5,6]$. Parts of

\footnotetext{
* Corresponding author: biancagomes@poli.ufrj.br
} 
commercial and high-strength Al alloys have been produced with TIG [7,8] and MIG [9-12] to exploit the full potential of the material as a printed part and reduce the occurrence of defects.

The pulsed GMAW has been used before in additive manufacturing [13], but not the pulse multi-control (PMC) and PMC Mix technology, to the best of the authors knowledge. The PMC and PMC Mix drive are pulse controlled spray arc optimized by fast control algorithms and should increase the process stability, improve the arc ignition, stabilize the deposit penetration, and reduce the heat input for the same deposition rate when compared to standard MIG and MIG Pulse. Also, the PMC Mix drive technology combines this pulse controlled transfer to cycles of controlled short circuit, a colder phase, reducing the heat input even more [14]. It is known PMC can enlarge the process window to keep the required mechanical properties when welding high strength steel, compared to standard MIG [15].

Cold Metal Transfer (CMT) is well known as an interesting controlled short circuit process because of its lower heat input, possible due to the mechanical reversal of the wire to facilitate the metal drop detachment, which allows the use of lower currents in the process. Also, variants of CMT, such as CMT-Pulse, which associates controlled short circuit to pulse cycles permitted the achieving of higher deposition rates [14]. Many parts have been produced with these technologies and the good results achieved in 2010 with Ti alloys [16], such as spatter free, low heat input, and reproducible deposits, motivated the study with other metals. Investigations with 2xxx [12,17], 4xxx [18], 5xxx series Al alloy [19] have also shown great potential when applying CMT and CMT-Pulse.

Aluminium alloys are recognized by their low specific density, good corrosion resistance, good ductility, and good formability [20]. Additions of $\mathrm{Mg}$ are made in $\mathrm{Al}$ alloys (5xxx series alloys) in order to improve their mechanical properties, so they can be used in the building sector and mechanical industries. Al-Mg alloys when combined with $\mathrm{Mn}$ and $\mathrm{Cr}$ also have outstanding corrosion resistance and are used in marine applications, naval construction, automotive industry, and industrial fabrication [21,22]. To achieve higher mechanical resistance and keep good ductility, they are also employed as cold worked [23], possible in additive manufacturing by rolling, for instance [19,24-26].

Therefore, in this study the additive manufacturing of the parts with ER5356 wire was performed and divided in two steps: (1) three different deposition strategies were used and the most suitable deposition strategy was defined as the one that produced the most regular part and (2) five different GMAW variants (CMT, CMT-Pulse, MIG Pulse, PMC, and PMC Mix) were used to produce the parts. The parts were then analysed by visual inspection and radiograph to identify surface and volumetric defects. Moreover, macrographs were obtained to check the presence of smaller defects and to count the porosities. Finally, hardness and tensile tests were performed to assess the mechanical properties of the components.

\section{Materials and methods}

\subsection{Additive manufacturing of samples}

The substrates used were $10 \mathrm{~mm}$ thick bars of AA6082 and the wire was ER5356 $1.2 \mathrm{~mm}$ in diameter used in as received condition. The chemical composition is presented in Table 1. The chemical analysis was performed using X-Ray Fluorescence technique with Niton XL2 GOLDD XRF. The substrate was brushed and cleaned with acetone just before the deposition starts. 
A Fronius TPS400i power source with CMT, CMT-Pulse, MIG Pulse, PMC, and PMC Mix droplet transfer modes were used for the parts building. In the first step of this study, CMT was used to deposit multi-layered boxes with 3 different trajectories: a) one-way discontinuous-arc, b) two-way discontinuousarc, and c) one-way continuous-arc, showed in Fig. 1. Also 3 different start points were used for trajectories (a) and (b) to attenuate the humps found at the beginning of the deposits and they are indicated in the Fig. 1 as P1, P2,

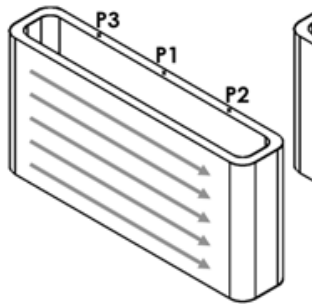

(a)

Fig. 1. Deposition strategies: (a) one-way discontinuous-arc; discontinuous-arc.

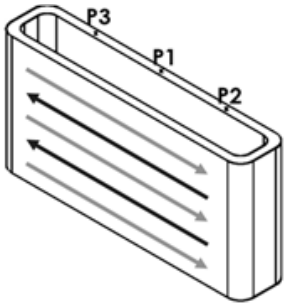

(b) and P3. CMT was used and the deposition parameters were $56 \mathrm{~A}$ (current - C), $12.7 \mathrm{~V}$ (tension - T), $4.1 \mathrm{~m} / \mathrm{min}$ (wire feed speed - WFS), and $60 \mathrm{~cm} / \mathrm{min}$ (travel speed - TS). The shielding gas used was Argon (99.99\%) with a flow rate of $15 \mathrm{l} / \mathrm{min}$ and the contact tip work distance was $15 \mathrm{~mm}$ for all the trials in this study.

Table 1. Chemical composition of substrate and wire (in wt.\%).

\begin{tabular}{|c|c|c|c|c|c|c|c|}
\hline & Si & Fe & Cu & Mn & Mg & Cr & Al \\
\hline AA6082 & 0.73 & 0.30 & 0.10 & 0.71 & 0.86 & - & 97.20 \\
\hline ER5356 & - & 0.11 & - & 0.13 & 4.40 & 0.13 & 95.20 \\
\hline
\end{tabular}

Multi-layered boxes were deposited layer by layer with one-way continuous-arc and 35 mm x $125 \mathrm{~mm}$ (width $\mathrm{x}$ length) dimension. Because the first layer of CMT presented a smaller WFS than the programmed one, higher WFS was used in the first three layers to ensure the proper fusion. In order to be able to compare CMT deposits with the other transfer modes all of the deposits were produced with higher WFS in the first three layers. Also, to evaluate CMT, CMT-Pulse, MIG Pulse, PMC, and PMC Mix droplet transfer modes, similar currents (differences up to $3 \mathrm{~A}$ ) and TS were kept for each GMAW variant. The deposition parameters used were 46 - 49 A (C), $12.1-16.1 \mathrm{~V}$ (U), $2.8-3.5 \mathrm{~m} / \mathrm{min}$ (WFS), and $60 \mathrm{~cm} / \mathrm{min}$ (TS).

\subsection{Sample testing and analysis}

The multi-layered boxes were evaluated by visual inspection and radiograph to verify the presence of defects and measured to check the geometric regularity in different sections. Then two transverse sections of the part were cut and mounted in resin, grinded with $\mathrm{SiC}$ paper, polished with diamond paste, and polished with colloidal silica. Finally, macrographs were obtained in the stereoscope (Olympus SZX7) to perform the

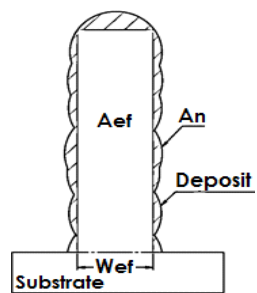

(a)

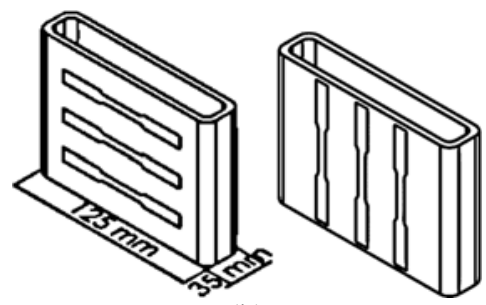

(b)

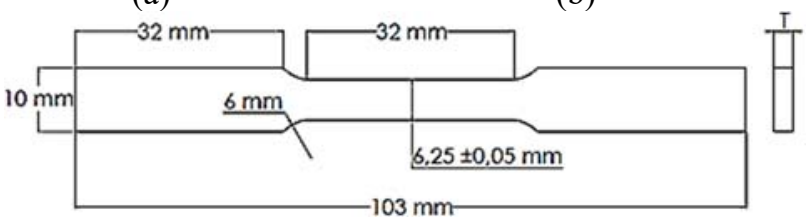

(c)
Fig. 2. (a) Illustration of measurements in transversal section of the boxes; (b) Longitudinal and transversal tensile test samples; (c) Drawing of the tension specimens. porosity counting and measures (ImageJ software), as showed in Fig. 2 (a). The effective 
width (Wef) is defined as the largest wall width achievable considering a flat wall surface and the null area (An) is the material would be removed in the post-processing step. Vickers hardness testing (hardness tester AVK-CO, Akashi Corporation) was made with a load of $1 \mathrm{kgf}$ and indentation time of $15 \mathrm{~s}$ along the transversal section. Uniaxial tensile tests were performed at room temperature by an electromechanical universal testing machine in specimens removed from two directions of the part, longitudinal and transversal to the deposition direction, Fig. 2 (b). The specimen test geometry was stablished in accordance with ASTM A370-16, Fig. 2 (c). The test was performed with a strain rate of $0,015 \mathrm{~min}^{-1}$ and the elongation after fracture was measured.

\section{Results and discussion}

\subsection{Deposition strategy}

It can be seen the parts produced, showed in Fig. 3, with one-way discontinuous-arc (part 1) and two-way discontinuous-arc (part 2) trajectories presented irregularities in their starting points. In the part 1, waviness is observed and in the part 2 an increment in the height of this region appears. In contrast, the other sides did not show irregularities in the height and surface finishing because of the well-adjusted deposition parameters and the regularity of the previous layers. According to investigators [27] the humps at the starting points are a consequence of the heat sink formed, which reduces the penetration of the arc. Similar behaviours would be expected in the building of geometries containing cross section for instance, in which if nothing was done the accumulation of material would be expected. Some researchers [2] presented the use of plasma-based WAAM equipment as a solution to this problem, since the control of WFS and C is independent. The part built with one-way continuous-arc trajectory (part 3), pictured in Fig. 3, is the most regular geometry, because there is just one starting point.

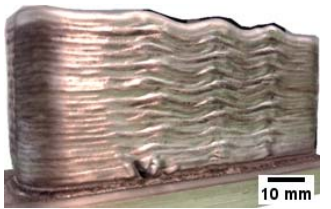

Part 1 - One-way discontinuous-arc

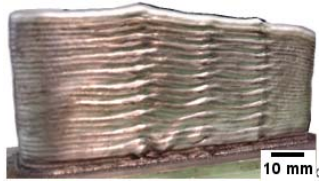

Part 2 - Two-way discontinuous-arc

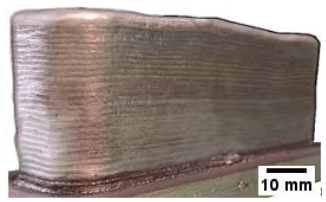

Part 3 - One-way continuous-arc

Fig. 3. Produced parts of 40 layers with CMT. C: 56 A; U: 12.7 V; WFS: $4.1 \mathrm{~m} / \mathrm{min}$; TS: $60 \mathrm{~cm} / \mathrm{min}$.

As can be seen in Fig. 4, it is noticeable that for part 1 the layer width increased and the deposit height decreased, when compared to part 3, due to the higher interpass temperature. Despite the fact the interpass temperature was not monitored by any device, the temperature increment is expected, since the weld arc is not interrupted until the end of the deposition. It is known the construction of large scale part would attenuate this effect due to the bigger mass to dissipate the heat and the higher time interval between layers. Another way to control the interpass temperature is using a cooling system in the substrate $[7,8]$. Furthermore, the reduction in the layer height, due to the higher interpass temperature, promoted an increase in the stick-out and instabilities in the metal transfer. In order to overcome this obstacle, a system was developed to control the height offset during the deposition of the layers.

Based on the results presented it was found the one-way continuous-arc trajectory assure better regularity in the part geometry, despite the reduction in the height. 


\subsection{Evaluation of the parts produced with variants of GMAW}

\subsubsection{Deposit uniformity}

30-layer parts were produced with CMT, CMT-Pulse, MIG Pulse, PMC, and PMC Mix, and their Wef, height, and An were compared (see Fig. 5 and 6). The first 3 layers were neglected in this case, since they were deposited with different parameters than the other ones. Considering the deposition parameters, it is seen the CMT granted a higher WFS for the same current range than the other transfer modes, which explain the higher height of the part. On the other hand, the height is not constant in the different sections measured, which causes more material waste in the post-processing step. Taking this into account, MIG-P and PMC show the smallest null area and very regular deposits, and thus, it can be affirmed they provided the best parts.

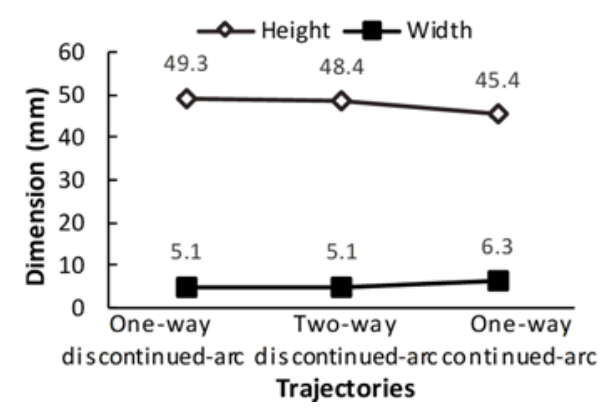

Fig. 4. Dimension of the 40-layer parts produced with different deposition trajectories.

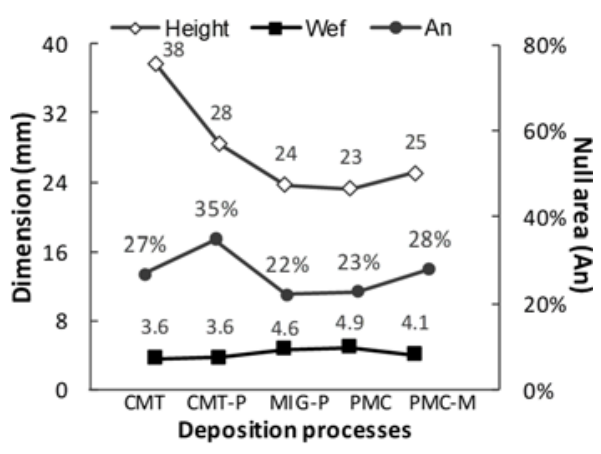

Fig. 5. Height, $W_{\text {ef, }}$ and $A_{n}$ of 30-layer parts produced with variants of GMAW.

The increasing in layer width along the height should be mentioned. It was found the layers tend to keep the same width, starting from the $6^{\text {th }}$ layer (approximately). Previous studies in the Ti6Al4V alloy deposited with WAAM, associated the width variation to the instantaneous heat conduction to the substrate and the high cooling rates. Since the heat dissipation to the atmosphere per convection and radiation is slower than the dissipation to a cool substrate, the heat concentrates in the weld pool resulting in a wider deposit [28].

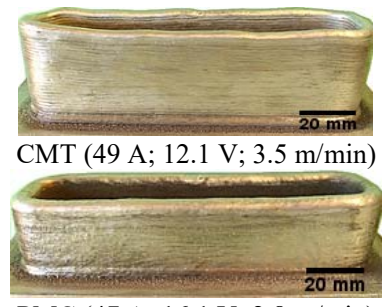

PMC (47 A; $16.1 \mathrm{~V} ; 2.8 \mathrm{~m} / \mathrm{min})$

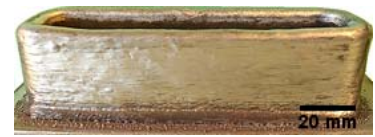

CMT-P (46 A; $15.8 \mathrm{~V} ; 3.1 \mathrm{~m} / \mathrm{min})$

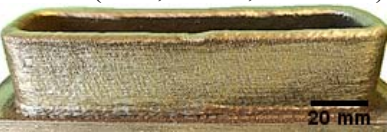

PMC-M (47 A; $14.8 \mathrm{~V} ; 2.7 \mathrm{~m} / \mathrm{min})$

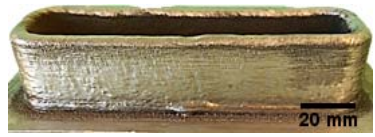

MIG-P (48 A; $15.4 \mathrm{~V} ; 2.8 \mathrm{~m} / \mathrm{min})$

Fig. 6. 30-layer parts deposited with variants of GMAW. Deposition parameters are cited in the following order: $\mathrm{C}, \mathrm{U}$, and WFS.

\subsubsection{Porosity of deposits}

The porosity in the 30-layer parts was evaluated based on the two macrographs of the whole transversal section. The pores are easily identified by bright and round spots and the parts representative areas analysed are $300-370 \mathrm{~mm}^{2}$ in total. The pore counts are presented in Fig. 7 (a). The arc transfer modes which resulted the smaller porosity fraction were PMC and 
MIG-P, see Fig. 7 (b). Also, all technologies presented porosity fraction smaller than $2 \%$, a pore fraction value associated to the reduction in tensile strength in the deposition of ER5356 parts with GMAW when compared to casted and wrought parts of the same material [29].

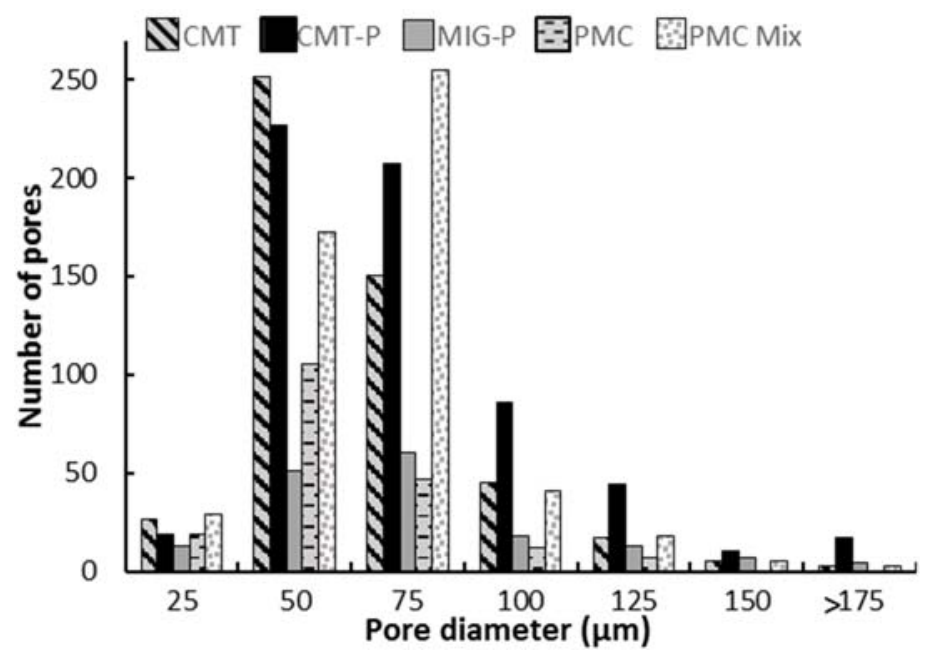

(a)

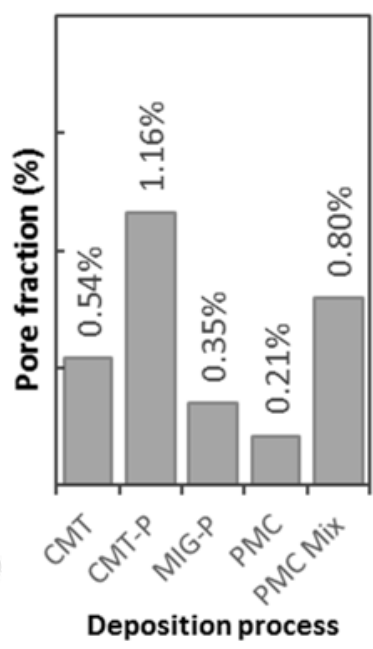

(b)

Fig. 7. Measurements obtained from 2 representative transversal sections of 30-layer parts produced with GMAW: (a) Histogram of pores; (b) Pore fraction.

\subsubsection{Hardness}

The hardness results obtained from the parts produced (see Fig. 8), indicate there is no significant difference among them. In this case, the hardness is directly related to the grain size (GS) and the secondary dendrite arm spacing (SDAS), being higher when GS and SDAS are smaller. The behaviour found in this study may be explained by similar GS and SDAS promoted by the variants of GMAW. Although the heat inputs are not the same for each technology used, the thermal cycle promoted by the subsequent layer may be responsible for these results. Investigators [30] related changes in hardness to the dendrite size found in AlMg deposits.

\subsubsection{Tensile tests}

The parts produced were firstly inspected by radiography and the results showed few small porosities, smaller than $1 \mathrm{~mm}$ diameter, dispersed in the samples. Tensile specimens were then obtained in the longitudinal and transversal to the deposition direction from parts with 120-150 layers. The results showed no significant difference can be noted when comparing the ultimate tensile strength (UTS) and yield strength (YS) found among the processes selected (see Fig. 9). Also the tensile properties were nearly isotropic with a difference of up to $13 \mathrm{MPa}$ higher in the longitudinal to the deposition direction. Furthermore, it was found a UTS slightly smaller in the deposited part when comparing with wrought parts of the same material, as can be seen in Table 2. This result can be associated to the porosities found in the component or to the amount of $\mathrm{Mg}$ in the alloy. Since only $4.4 \%$ of $\mathrm{Mg}$ was found in the deposit it might have affected the UTS of the alloy [32]. 


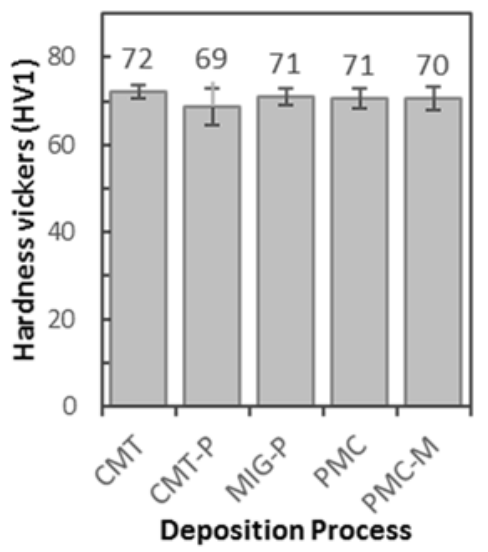

Fig. 8. Hardness of 30-layer parts produced with variants of GMAW.

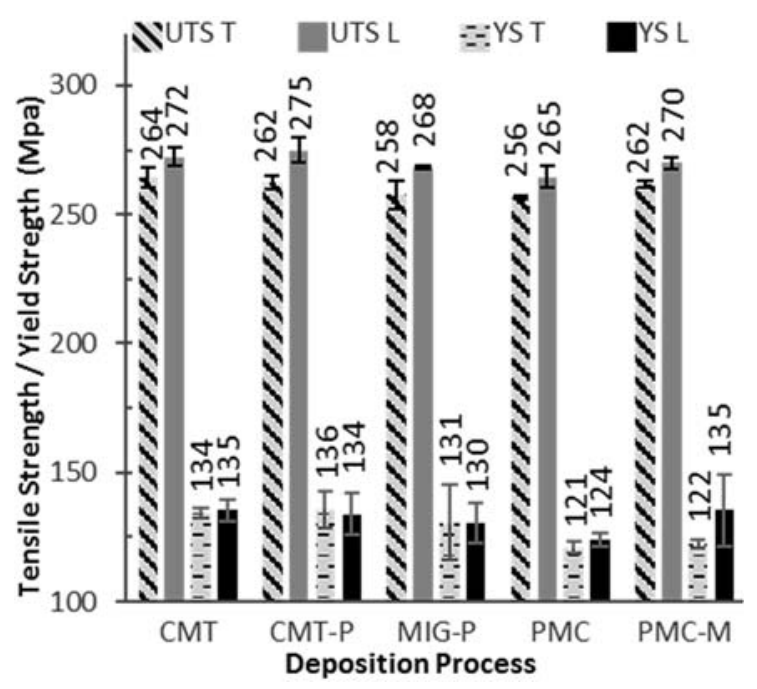

Fig. 9. Ultimate tensile strength (UTS) and yield strength (YS) of the parts produced with variants of GMAW.

Table 2. Mechanical properties of Al alloy 5356 as wrought and as deposited.

\begin{tabular}{|c|c|c|c|c|c|}
\hline Alloy & Process & Yield Strength (MPa) & UTS (MPa) & Elongation (\%) & Reference \\
\hline AA5356 & Wrought & 130.0 & 285.0 & - & {$[31]$} \\
\hline ER5356 & Deposited & $130.2 \pm 8.6$ & $265.2 \pm 6.4$ & $32.8 \pm 3.8$ & This study \\
\hline
\end{tabular}

\section{Conclusion}

Components produced with one-way continuous-arc trajectory presented better regularity in the part geometry because of the unique starting point imposed in this case. When using other trajectories, humps are formed affecting the regularity of the deposits. Optimized parameters control could attenuate or even eliminate this obstacle.

When considering the CMT, CMT-Pulse, MIG Pulse, PMC, and PMC Mix processes applied to ER5356 alloy, it was found they lead to very similar mechanical properties when using similar deposition parameters. Although CMT presented the highest deposition rate it also presented very different height along the part produced, resulting in a non-uniform part. It happened mainly because of the WFS variation characteristic of this process. Lastly, the MIG-P and PMC showed the smaller pore fraction even though all deposits produced with GMAW variants presented small pore fraction and small pores dispersed in the parts.

Thus, based on the good mechanical properties, small pore fractions, and regularity of deposits obtained with PMC and PMC Mix even when compared with CMT, well known for its good results in additive manufacturing, these technologies should be further exploited. Deposition parameters shall be optimized and higher deposition speeds shall be used to obtain the combination of few defects and good productivity.

\section{References}

1. D. Brackett, I. Ashcroft, R. Hague, Proc. solid Free. Fabr. Symp. 1, 348-62 (2011)

2. P.A. Colegrove, A.R. McAndrew, J. Ding, F. Martina, P. Kurzynki, S. Wiliams, 10th Int. Conf. on Trends in Weld. Res. (2016) 
3. J.S. Panchagnula, S. Simhambhatla, Virtual Phys Prototyp. 11, 99-108 (2016)

4. F. Martina, Doctoral thesis, Cranfield University (2014)

5. F. Wang, S. Wiliamns, P. Colegrove, A. Antonysamy, Metall Mater Trans A Phys Metall Mater Sci 44, 968-77 (2013)

6. D. Ding, D. Cuiuri, Rob. Comp.-Integ. Manuf. 31, 101-110 (2015)

7. H. Geng, J. Li, J. Xiong, X. Lin, F. Zhang, J Mater Process Technol 243, 40-7 (2017)

8. K.F. Ayarkwa, S. Wiliams, J. Ding, Addit. Manuf. 18, 186-93 (2017)

9. P. Wang, S. Hu, J. Shen, Y. Liang, J Mater Process Technol 245, 122-33 (2017)

10. H. Liu, S. Yang, C. Xie, Q. Zhang, Y. Cao, J Alloys Compd 741, 188-96 (2018)

11. A.S. Haselhuhn, E.J. Gooding, A.G. Glover, G.C. Anzalone, B. Wijnen, P.G. Sanders, 3D Print Addit Manuf 1, 204-9 (2014)

12. B. Cong, J. Ding., S. Wiliams, Int J Adv Manuf Technol 76, 1593-606 (2015)

13. F. Wang, S. Wiliams, M. Rush, Int J Adv Manuf Technol 57, 597-603 (2011)

14. Fronius. https://www.fronius.com/en (accessed in August 3, 2018)

15. M. Fiedler, A. Plozner, B. Rutzinfer, Biul Inst Spaw w Gliwicach 60, 31-7 (2016)

16. P.S. Almeida, twenty-first Annu. Int. solid Free. Fabr. Symp., Univ. Texas Austin (2010)

17. X. Fang, L. Zhang, H. Li, C. Li, K. Huang, B. Lu, Materials 11, 812 (2018)

18. A.G. Ortega, L.G. L.C. Galvan, F.D. Beaume, Sci Technol Weld Join 23, 316-22 (2018)

19. J. Gu, X. Wang, J. Bai, J. Ding, S. Williams, Y. Zhai, Mater Sci Eng 712, 292-301 (2018)

20. I. Hrivñák, Theory of weldability of metals and alloys (1992)

21. G. Mathers, The welding of aluminium and its alloys (2002)

22. C. Vargel, Corrosion of Aluminium (2004)

23. J.E. Hatch, Aluminum Properties and Physical Metallurgy (1984)

24. P. Colegrove, F. Martina, M. Roy, B. Szost, Adv. Mat. Res. 996, 694-700 (2014)

25. F. Martina, P. Colegrove, S. Williams, J. Meyer, Met. Mat. Trans. A 46, 6103-18 (2015)

26. F. Martina, M. Roy, B. Szost, S. Terzi, et al Mater Sci Technol. 32, 1439-1448 (2016)

27. Y.M. Zhang, Y. Chen, P. Li, A.T. Male, J Mater Process Technol 135, 347-57 (2003)

28. B. Wu, D. Ding, Z. Pan, D. Cuiuri, H. Li, J. Han, J Mat. Proc. Techn. 250, 304-12 (2017)

29. A. Haselhuhn, M. Buhr, B. Wijnen, Sanders, Pearce, Mater Sci Eng 673, 511-23 (2016)

30. A. Haselhuhn, B. Wijnen, G. Anzalone, Mater. Process. Technol. 226, 50-59 (2015)

31. J.G. Kaufman, Prop. of Al alloys: tensile, creep, and fatigue data at high and low (1999)

32. L. F. Mondolfo, Aluminum alloys: structure and properties (2013) 